\section{Assessment of the AnyBody Full Body Musculoskeletal Model in Computing Spine Loads at Lumbar Level: Comparison with in vivo Values Obtained during Exercise Tasks}

\author{
Tito Bassani', Elena Stucovitz, Zhihui Qian², Matteo Briguglio and Fabio Galbusera ${ }^{1}$ \\ 1 IRCCS Istituto Ortopedico Galeazzi, Milan, Italy (www.labsgaleazzi.it) \\ ${ }^{2}$ Key Laboratory of Bionic Engineering, Jilin University, Changchun, P.R. China
}

\title{
INTRODUCTION
}

Musculoskeletal models provide a valuable insight to non-invasively investigate the relation between human motion and internal biomechanical loads. Through an inverse dynamic approach, this modeling technique allows to compute the intersegmental forces acting during the execution of imposed specific body motion and under the action of known external loads. When focusing on the characterization of human spine, musculoskeletal modeling can be accounted to evaluate lumbar loads during physiological activities (e.g. training, ergonomics and rehabilitation) and pathological scenarios (e.g. spine deformities and surgical fixation strategies). In the panorama of available modeling software, AnyBody software is a powerful commercial tool that provides a full body musculoskeletal model, used by several researchers worldwide. When exploited to predict lumbar loads, the previous works aimed at validating model suitability held several limitations [1-3] and a comprehensive validation is to be considered as lacking. The present study was thus aimed at validating the suitability of the AnyBody model in computing lumbar loads at L4L5 level in physiological conditions.

\section{METHODS}

One healthy male subject (28 years) was enrolled in the study. According to VICON protocol, 41 passive markers were placed on the skin (Fig.1a,b). Motion capture data were acquired during the execution of specific tasks and then imported into AnyBody to set model kinematics (Fig.1c,d).

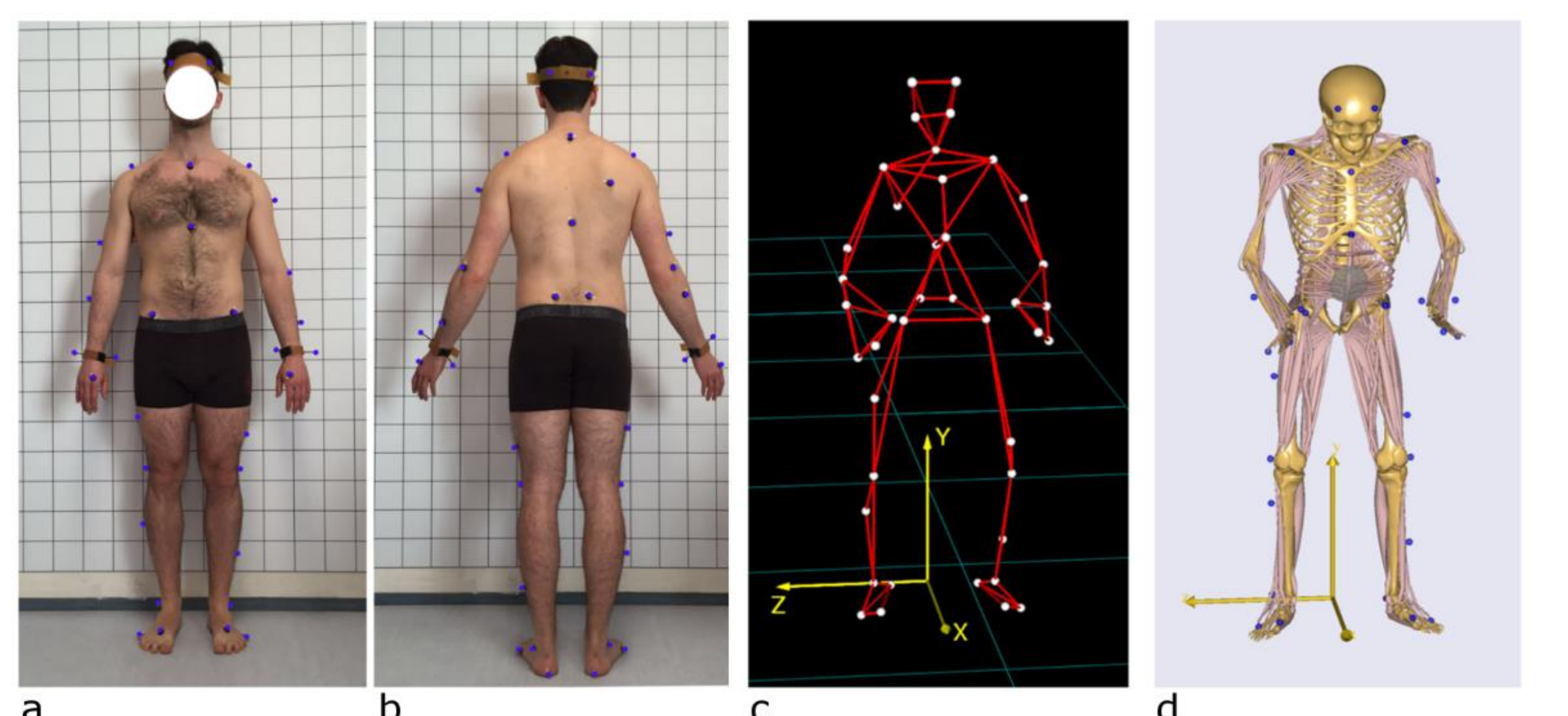

Figure 1: Front (a) and back (b) view of the acquired subject with the 41 passive markers placed on the skin, and markers positions in the 3D space acquired with motion capture (c) and subsequently imported to set the AnyBody mannequin kinematic (d).

Twelve exercise tasks (Table1) were performed to accurately replicate the corresponding exercise conditions during which Wilke et al. [4] evaluated in vivo the L4L5 disc nucleus pressure. The intersegmental axial load at L4L5 level was calculated with the body model for each task. During flexionextension, lateral bending and axial rotation tasks the L4L5 loads were evaluated in dynamic continuous fashion in dependence on the angle between the thoracolumbar junction (T12) and the sacrum.

Table. 1: The twelve exercise tasks performed to replicate those evaluated by Wilke et al. [4] and corresponding postures of the AnyBody full-body mannequin.

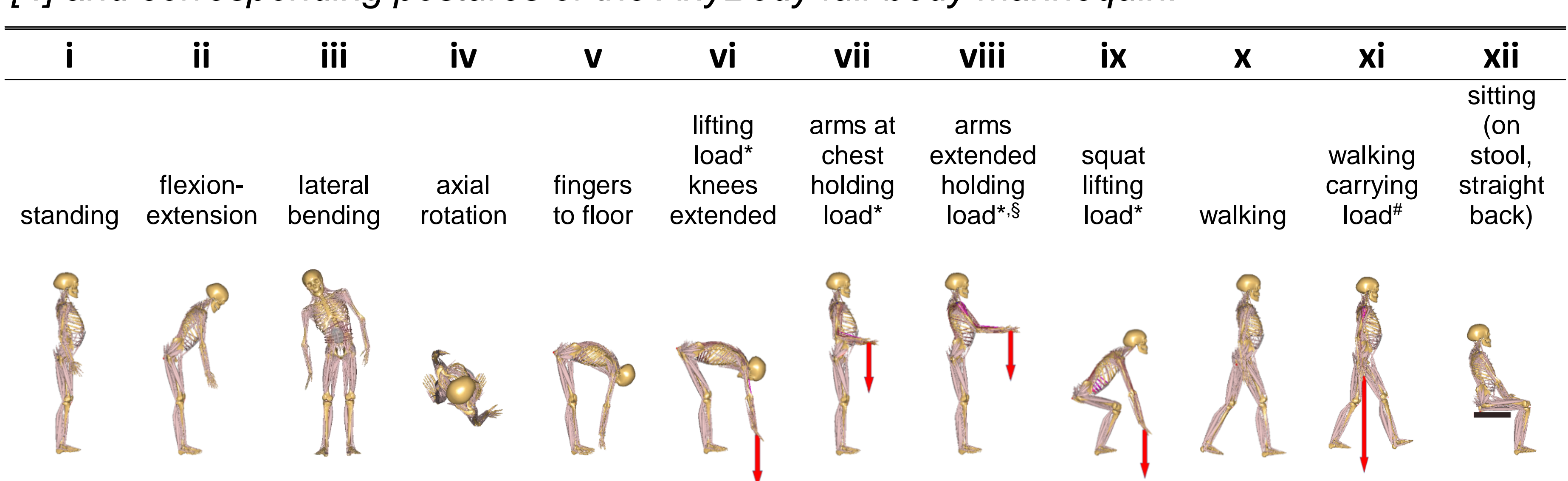

", lifting with both hands a barbell loaded at the center with $20 \mathrm{~kg}$, in order to replicate the lifting of a crate of beer of $19.8 \mathrm{~kg}$ of weight used in Wilke et al. [4].

\#, carrying with the right hand a dumbbell loaded with $20 \mathrm{~kg}$.

, barbell was held $60 \mathrm{~cm}$ away from chest, according to Wilke et al. [4].

The corresponding L4L5 average pressure was derived accounting for disc area. The disc nucleus pressure was obtained from the average pressure according to two different approaches: correction factor (CF) based on in vitro study [5], and quadratic equation (QE) based on in silico study [6]. The computed pressures were compared with the in vivo measurements.

\section{DISCUSSION}

Positive agreement between the calculated values and the in vivo measurements was generally confirmed during all tasks (Fig.2). Significant very strong Pearson correlation coefficient was pointed out using both the CF and QE approaches (0.91 and 0.90 , respectively). Generally, the pressure values calculated with CF resulted from 0.1 to $0.2 \mathrm{MPa}$ higher than those computed with QE.

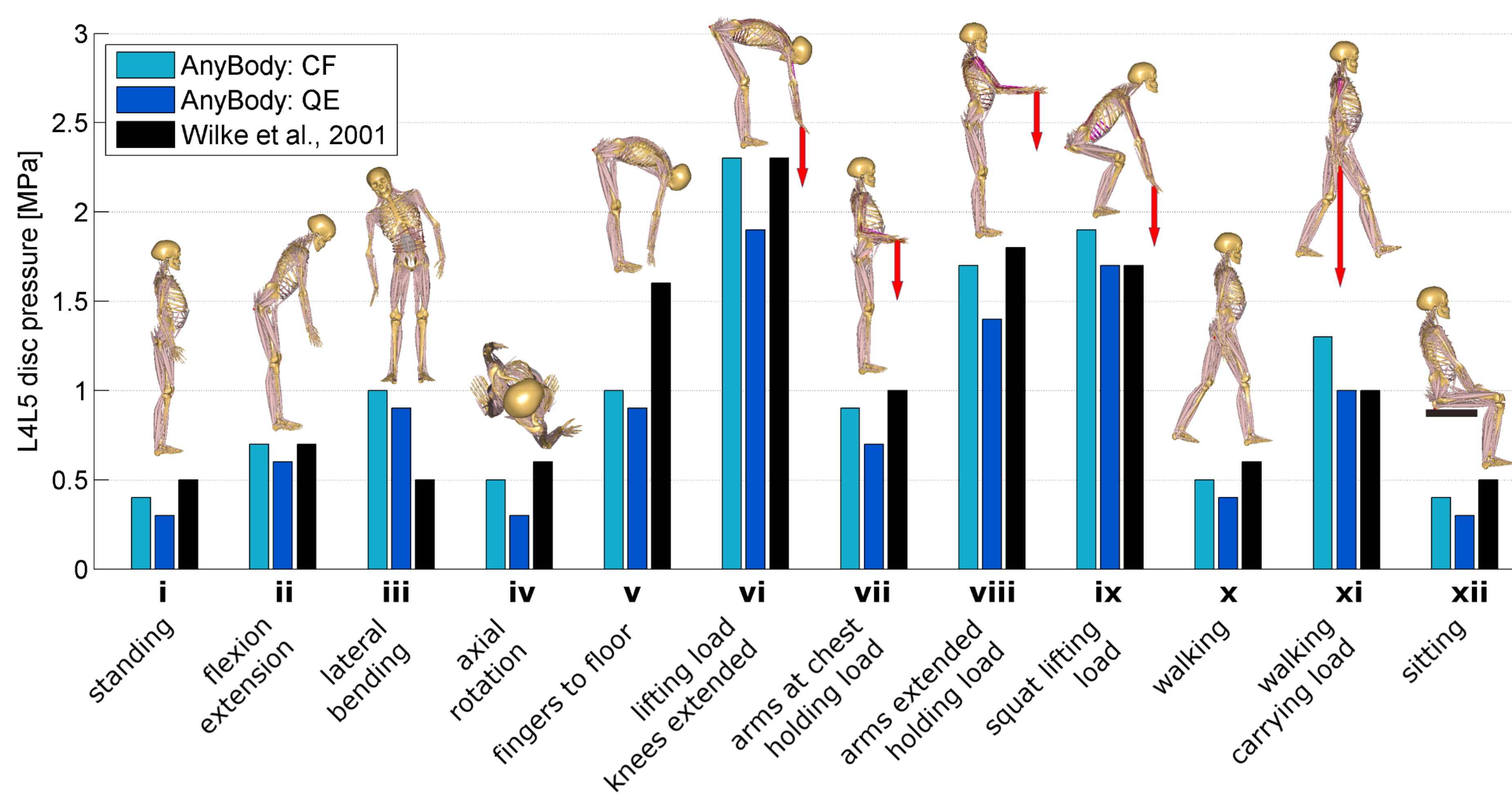

Figure 2: L4L5 disc pressure calculated with AnyBody model according to CF and QE approaches (presented in light blue and dark blue, respectively) and measured in vivo by Wilke et al. [4] (presented in black) in the specific task.

When assessing the relationship between disc pressure and body motion angles, comparable values were exhibited during flexion-extension and axial rotation, whereas differences were found in lateral bending when the angle between the thoracolumbar junction end the pelvis exceeded $\pm 15^{\circ}$ (Fig.3). The results of the present work demonstrate the suitability of the AnyBody model in computing lumbar spine loads at L4L5 level. Specific caution needs to be taken only when considering postures characterized by

\section{References}

[1] de Zee, M. et al., 2007. A generic detailed rigid-body lumbar spine model. J. Biomech. 40:1219-1227.

2] Rasmussen, J., et al., 2009. Validation of a biomechanical model of the lumbar spine. XXIInd Congress of the International Society of Biomechanics.

3] Rajaee, M.A. et al., 2015. Comparative evaluation of six quantitative lifting tools to estimate spine loads during static aliviles. Appl. Ergon. 48:22-32.

(4) validation of models. . Biomech. (Bistol, Avon) 16 Suppl 1:S111-26. large lateral displacements or high lateral loading. The present findings promote the AnyBody model as an appropriate tool to non-invasively evaluate lumbar loads in physiological activities. Future studies can be aimed at evaluating the use of AnyBody modeling in pathological conditions known altering spine alignment, such as spine deformities and fixation strategies.

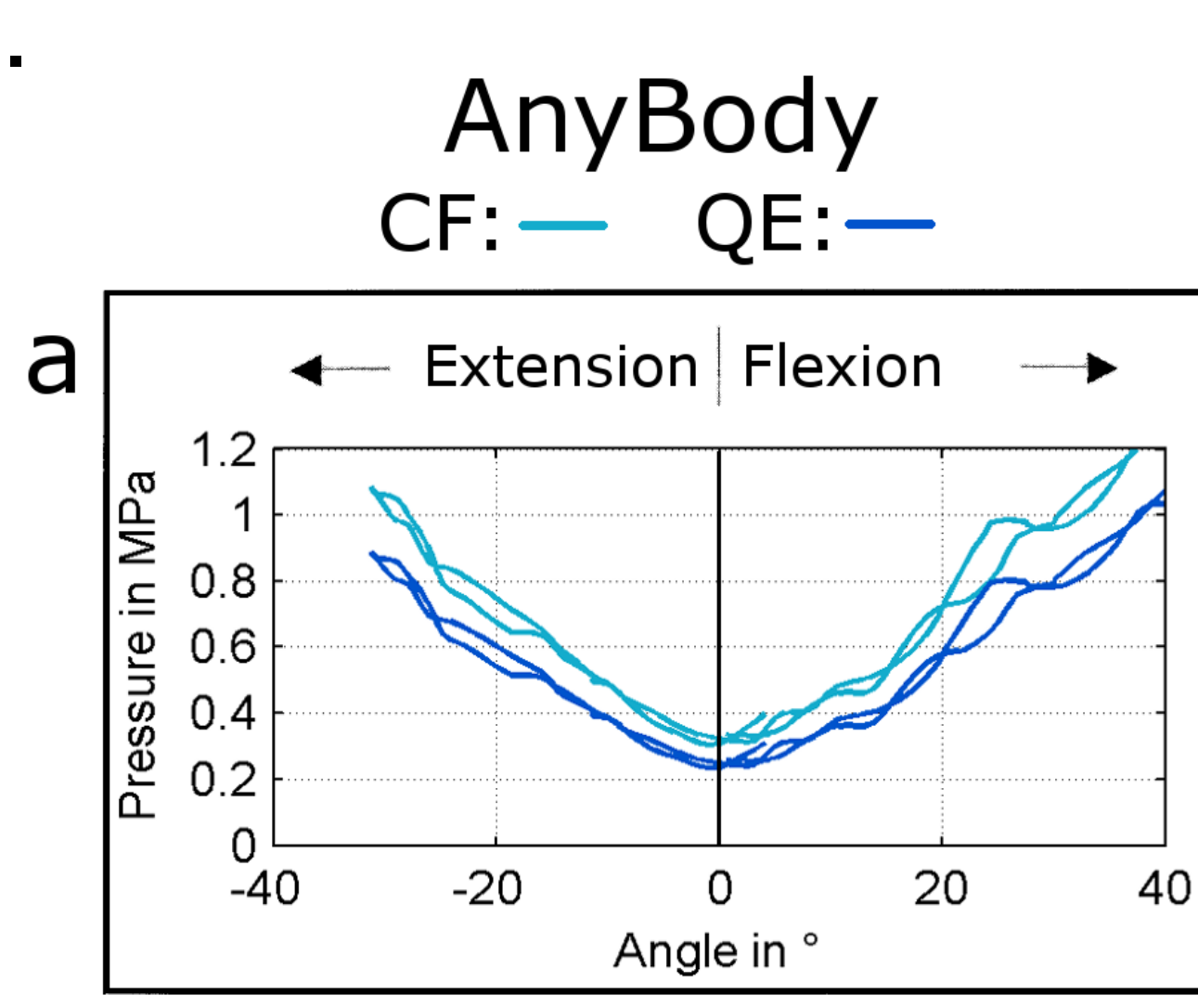

Wilke et al., 2001
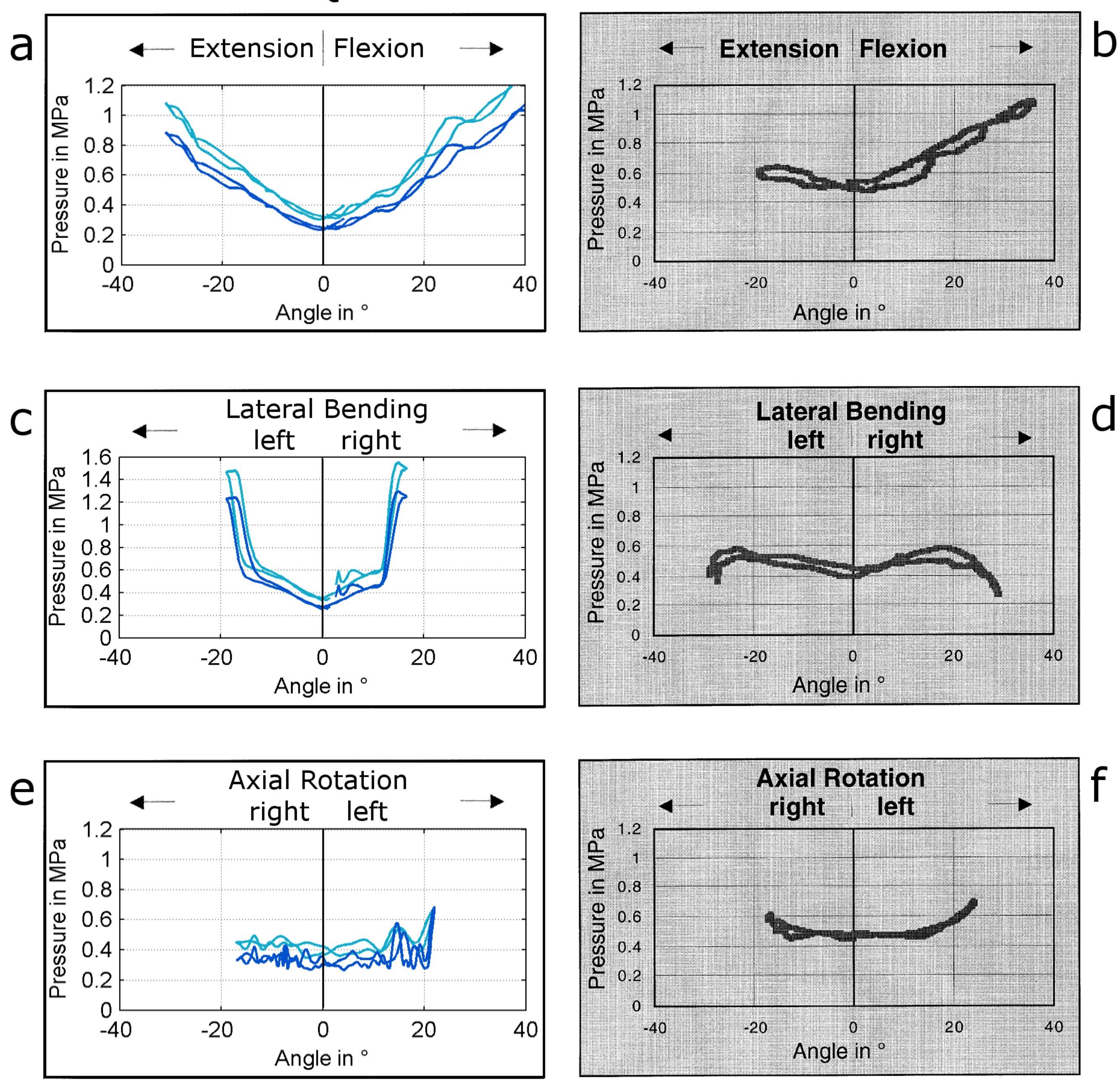

Figure 3: $L 4 L 5$ disc pressure in dependence on the angle between the thoracolumbar junction and the sacrum, calculated with AnyBody model (left column) according to CF and QE approaches (depicted in light blue and dark blue, respectively) and measured in vivo by Wilke et al. [4] (right column) during flexion-extension (a,b), lateral bending $(c, d)$ and axial rotation tasks $(e, f)$

[5] Brinckmann, P. et al., 1991. Change of Disc Height, Radial Disc Bulge, and Intradiscal Pressure from Discectomy: An In Vitro Investigation on Human Lumbar Discs. Spine (Phila Pa 1976) 16(6):641-646.

[6] Ghezelbash F. et al., 2016. Subject-specific biomechanics of trunk: musculoskeletal scaling, internal loads and intradiscal pressure estimation. Biomech. Model. Mechanobiol. 15(6):1699-1712.

Acknowledgements

The study was fully supported by the Italian Ministry of Foreign Affairs and International Cooperation (Project 